\title{
Paired comparison scaling of reaction potential from simultaneous measurements of GSR and looking time
}

\author{
H. D. KIMMEL, UNIVERSITY OF SOUTH FLORIDA \\ CAROL BOICE, OHIO UNIVERSITY \\ BRUCE T. LECKART, 1 SAN DIEGO STATE COLLEGE
}

Thurstone's method of scaling from paired comparison data was employed to obtain estimates of reaction potential on eight successive presentations of randomly generated polygons. One set of scale values was based upon the magnitude of the GSR made by each of $40 \mathrm{Ss}$ to the onset of each stimulus. Another set of scale values was based upon the Ss' looking times, when the $S$ was instructed to press a switch to terminate the stimulus after he finished looking at it. Following termination of each stimulus, a 20-sec period of no stimulation intervened prior to the next stimulus. The GSR reduced in size from trial to trial, describing a typical habituation curve, while looking time increased from trial to trial. Across trials the two sets of scale values were significantly negatively correlated $(r=-0.91)$. The failure to find habituation of looking time was explained on the assumption that the 20-sec period between stimuli was noxious to the Ss, which resulted in longer and longer looking times across trials. These results were interpreted as indicating that habituation of the GSR is not merely a matter of reduced attention in the cognitive sense. In addition, it was pointed out that the use of orienting reflex concepts in dealing with attentional and other cognitive phenomena may be unjustified.

The principal contribution of Hull, Felsinger, Gladstone, and Yamaguchi's (1947) extension of Thurstone's paired comparison scaling procedure to behavioral studies is the fact that it provides an operational definition of reaction potential which is independent of the response units actually employed in any particular experiment. As Kimmel, Boice, and Leckart (in press) have recently illustrated, this technique permits comparisons to be made between different experimental representations of allegedly similar processes, even when entirely different arbitrary measurement units are involved. In the Kimmel, Boice, and Leckart example, reaction potential scaled from looking time, measured in seconds, and reaction potential scaled from the GSR, measured in change in conductance, were shown to decline across repeated stimulations in similar fashions, except for the fact that a more rapid drop from the first to the second stage characterized the GSR scale values as compared with the looking time values.

Since the GSR scale values were based on single-trial measurements while the looking time scale values were based on blocks of eight trials, the possibility existed that single-trial measures of both responses would result in scale values that reflected even more similar habituation processes. The purpose of the present study, thus, was to obtain simultaneous GSR and looking time measures from the same Ss, during an experimental procedure calculated to result in common habituation processes in both responses. Because of the long latency of the GSR and its vulnerability to elicitation by overt muscular reactions, it was necessary to alter the standard looking time procedure in such a way that the response which terminated a stimulus did not result in immediate presentation of the subsequent stimulus. When the $\mathrm{S}$ terminated a stimulus by pressing his switch, a 20 -sec delay intervened before the automatic presentation of the next stimulus, so that a GSR could be elicited by stimulus onset per se.

\section{METHOD}

Subjects

Forty undergraduates at Ohio University volunteered to be Ss to meet an introductory psychology class requirement. All of the Ss received the same conditions.

\section{Apparatus}

The GSR was picked up as a $\mathrm{dc}$ resistance change, transformed to values of conductance change, by Beckman skin electrodes. The S's hand was first cleaned with acetone. The response was amplified and recorded on a Grass Model 5 polygraph (5P-1 preamplifier), with a paper speed of $10 \mathrm{~mm} / \mathrm{sec}$.

The stimuli were eight 18-sided randomly generated polygons, constructed according to Method 1 outlined by Attneave and Arnoult (1956). The internal portions of the figures were black and the background white. They were photographed on Kodak high contrast positive copy film and mounted in $2 \times 2$ in. metal slide mounts. The stimuli were projected on a white lenticular screen by a Kodak Carousel 700 projector placed $12 \mathrm{ft}$ from the screen. The $\mathrm{S}$ sat in a chair $6 \mathrm{ft}$ from and facing the screen, on which a $20 \times 30$ in. image was projected. The stimuli were terminated with a manual button switch held in the S's right hand. Stimulus onset and looking time were automatically recorded to the nearest $0.1 \mathrm{sec}$ by the event recorder in the Grass polygraph.

\section{Procedure}

Data were collected in a sound-attenuated room. The GSR electrodes were attached to the palm and back of the S's left hand and $S$ was told that the purpose of the experiment was to monitor his GSR to visual stimuli. He was urged to avoid unnecessary or abrupt movements. The stimuli were presented automatically and the $S$ was instructed that when he finished viewing a stimulus he should terminate it by pressing the switch. The termination of each stimulus was followed by a $20-\mathrm{sec}$ period during which the screen was blank but

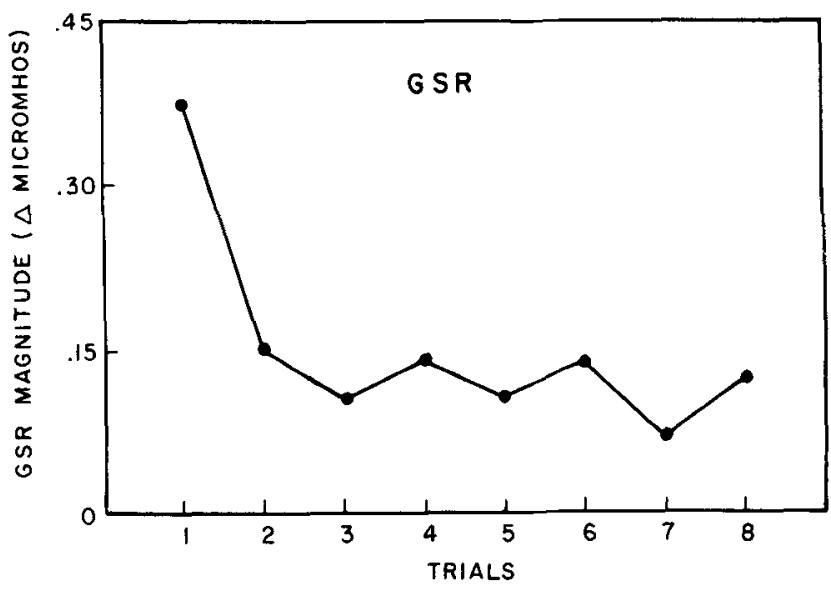

Fig. 1. Average magnitude of the GSR ( $\Delta$ micromhos) on eight trials. 


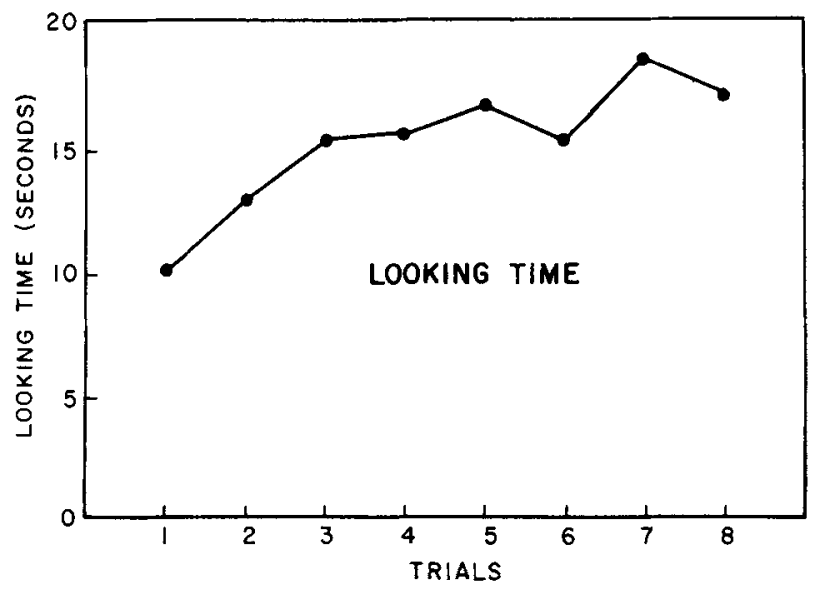

Fig. 2. Average looking time (seconds) on eight trials.

illuminated. The next stimulus was automatically projected at the end of the 20 -sec period. Each $S$ viewed the stimuli in a separately randomized order.

\section{RESULTS}

Figures 1 and 2 present the average GSR magnitudes and average looking times, respectively, on each of the eight trials. Each response is expressed in its own arbitrary units in these graphs. Inspection of Figs. 1 and 2 reveals that the GSR showed a smooth decline in response strength, typical of habituation, while looking time displayed a somewhat surprising continuous increase across the eight trials.

The details of the scaling procedure have been presented previously (Kimmel, Boice, \& Leckart, in press). Basically, a proportion matrix is constructed with consecutive trials on each axis, such that the value in each cell represents the proportion of Ss whose response on the trial number of that column was larger (or longer) than the response on the trial number of that row. A proportion of 0.50 is entered in those cells involving the comparison of a response with itself. Tables 1 and 2 show the proportion matrices for the GSR and looking time data, respectively.

The proportions in Tables 1 and 2 are then converted into normal-deviates, $z$, representing the value on the abscissa of the unit normal curve which cuts off that cumulative proportion of the area above and to the left of it. Under Thurstone's Case V assumptions, the averages of these normal deviates, or scale separations, would be taken as the scale values. However, even if the standard deviations of the hypothetical underlying normal distributions of behavioral oscillation could be assumed to be homogeneous across trials within the GSR and looking time data separately, it would be unjustified to assume homogeneity between the two different sets of measures. Thus, it is desirable to weight the obtained scale separations by multiplying them by estimates of $\sqrt{\sigma_{1}^{2}+\sigma_{2}^{2}}$, using the estimation method suggested by Burros (1951). Thus, Thurstone's less stringent Case III assumptions

Table 1

Ptoportion Matrix based on GSR Magnitude Data

\begin{tabular}{|c|c|c|c|c|c|c|c|c|c|}
\hline \multirow{5}{*}{ 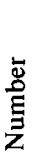 } & & \multicolumn{8}{|c|}{ Trial Number } \\
\hline & & 1 & 2 & 3 & 4 & 5 & 6 & 7 & 8 \\
\hline & 1 & .500 & .262 & .088 & .150 & .112 & .138 & .050 & .062 \\
\hline & 2 & .738 & .500 & .412 & .425 & .362 & .412 & .312 & .388 \\
\hline & 3 & .912 & .588 & .500 & .475 & .450 & .412 & .338 & .375 \\
\hline .尹̈ & 4 & .850 & .575 & .525 & .500 & .500 & .500 & .475 & .450 \\
\hline$\Xi$ & 5 & .888 & .638 & .550 & .500 & .500 & .512 & .512 & .425 \\
\hline & 6 & .862 & .588 & .588 & .500 & .588 & .500 & .462 & .475 \\
\hline & 7 & .950 & .688 & .662 & .525 & .488 & .538 & .500 & .462 \\
\hline & 8 & .938 & .612 & .625 & .550 & .575 & .525 & .538 & .500 \\
\hline
\end{tabular}

Table 2

Proportion Matrix based on Looking Time Data

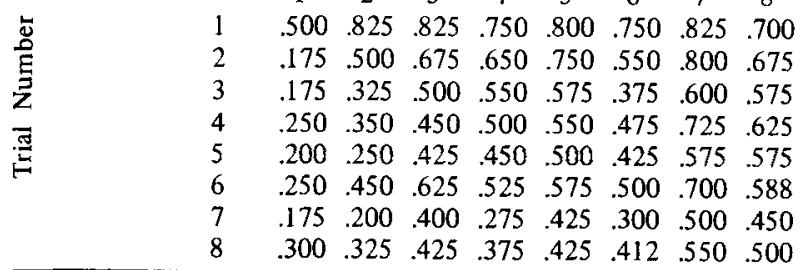

are involved. Tables 3 and 4 present the weighted scale separations and, in the next to the last row, the average scale values. In the bottom row a constant is added to the scale values to eliminate negative numbers. (The constant used in each case equals the absolute value of the most negative quantity.)

Figure 3 presents the GSR and looking time scale values, plotted on a common ordinate. It is quite clear in this graph that the looking time measures did not manifest anything even remotely resembling what would commonly be called habituation, while the GSR generated scale values of reaction potential describing a very smooth negatively accelerated decline.

Mosteller's (1951) method for assessing the internal consistency of the obtained scale values indicated that neither set of values approached significant inconsistency (GSR: $p>0.99$; Looking time: $p \cong 0.90$ ). The product moment correlation between the GSR and looking time scale values was -0.91 , which was highly significant even with $\mathrm{df}=6$.

\section{DISCUSSION}

The purpose of this study was to compare trial-by-trial changes in reaction potential scaled from GSR and looking time measures which were simultaneously collected in an habituation situation. It was assumed in advance that both sets of values would decline across trials in a way characteristic of habituation. Obviously, as is shown in Fig. 3, one of the measures (GSR) showed typical habituation across trials while the other (looking time) showed quite the opposite trial-by-trial pattern of change $(r=-0.91)$.

Since the GSR data nicely replicate the findings of several prior studies of habituation of this response (e.g., Kimmel, Boice, \& Leckart, in press), there is little reason to doubt that the current procedure of presenting a series of randomly generated polygons provided an opportunity for the

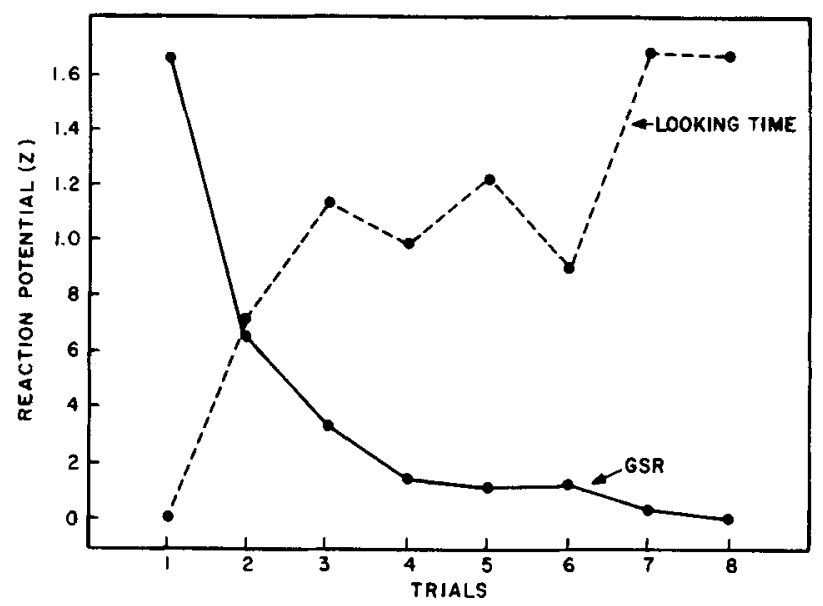

Fig. 3. Scaled values of reaction potential based on GSR and looking time. 
Table 3

Scale Separations between Pairs of GSR Trials $\left(\mathrm{z}_{\mathrm{jk}} \sqrt{ } \sigma_{\mathrm{j}}^{2}+\sigma_{\mathrm{k}}^{2}\right)$

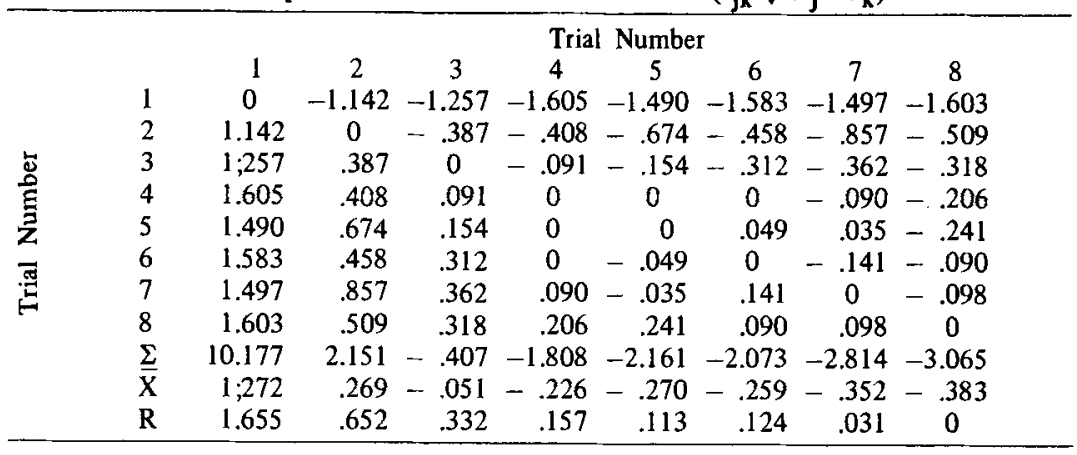

Table 4

Scale Separations between Pairs of Looking Time Trials $\left(\mathrm{z}_{\mathrm{jk}} \sqrt{ } \sigma_{\mathrm{j}}^{2}+\sigma_{\mathrm{k}}^{2}\right)$

\begin{tabular}{|c|c|c|c|c|c|c|c|c|c|}
\hline \multirow{13}{*}{ 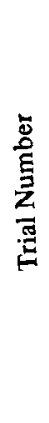 } & \multicolumn{9}{|c|}{ Trial Number } \\
\hline & & 1 & 2 & 3 & 4 & 5 & 6 & 7 & 8 \\
\hline & 1 & 0 & 1.104 & 1.211 & .896 & 1.165 & .959 & 1.382 & 1.385 \\
\hline & 2 & -1.104 & 0 & .341 & .318 & .600 & .125 & .875 & 1100 \\
\hline & 3 & -1.211 & -.341 & 0 & .127 & .198 & -.469 & .293 & .475 \\
\hline & 4 & -.896 & -.318 & -.127 & 0 & .141 & -.069 & .727 & .807 \\
\hline & 5 & -1.165 & -.600 & -.198 & -.141 & 0 & -.228 & .240 & .484 \\
\hline & 6 & -.959 & -.125 & .469 & .069 & .228 & 0 & .683 & .566 \\
\hline & 7 & -1.382 & -.875 & -.293 & -.727 & -.240 & -.683 & 0 & -.338 \\
\hline & 8 & -1.385 & -1.100 & -.475 & -.807 & -.484 & -.566 & .338 & 0 \\
\hline & $\underline{\Sigma}$ & -8.102 & -2.255 & .928 & -.265 & 1.608 & -.931 & 4.538 & 4.479 \\
\hline & $\overline{\mathbf{X}}$ & -1.013 & -.282 & .116 & -.033 & .201 & -.116 & .567 & .560 \\
\hline & $\mathrm{R}$ & 0 & .731 & 1.129 & .980 & 1.214 & .897 & 1.580 & 1.573 \\
\hline
\end{tabular}

autonomic component of the orienting reaction to habituate. What must be explained is the unexpected increase in looking times across trials. Although single-trial renditions of looking times do not appear in the published literature (since it is common for blocks of trials to be employed), a decrement in looking times over trial blocks normally occurs in studies employing standard looking time procedures (e.g., Leckart, 1968). While it is possible that even in standard looking time experiments an increase might occur over the very early trials (say, the first three or four), it is unlikely that this would continue for as many as eight triais, as occurred in the present experiment. Thus, it seems quite possible that the introduction of a 20-sec blank period between stimuli, which was done to permit uncontaminated measurement of the GSR, was responsible for the unusual looking time results. Assuming that this period without a stimulus may have been a noxious event relative to those periods when a stimulus was present, it is not entirely surprising that the Ss would gradually come to delay termination of the stimulus and initiation of the blank period. Viewed in this way, the looking time data reflect a kind of punishment phenomenon, with the punished response being the operation of the switch that terminated the stimulus. The latency of this response steadily increased across trials. Obviously, this post hoc conjecture could be checked most directly by collecting single-trial looking time data under varying amounts of delay between the termination response and the onset of the next stimulus.

Even with unexpected results, this study has two potentially important implications. Many investigators who have studied attentional behavior (e.g., Berlyne, 1958), tend more and more to employ the concepts and terminology of the orienting reflex laboratories. But this language is based upon the study of reactions which are largely of the vegetative and involuntary class, such as cortical evoked potentials, the vasomotor reflex, and the GSR. The results of the present study illustrate the danger inherent in the naive assumption that an instructed, cognitively mediated response, such as is reflected in looking time, can be comprehended in a framework built upon observations of autonomic or cortical psychophysiological reactions. In addition, habituation of the GSR in this experiment was quite clearly not merely a cognitive matter of reduced attention or boredom, since looking time increased at the same time that the GSR decreased over trials. Thus, even while the $S$ may have been adapting to the cognitive aspect of the situation by viewing the stimuli for longer and longer times, the nonadaptive orienting reflex (Sokolov, 1963) simply habituated automatically.

\section{REFERENCES}

ATTNEAVE, F., \& ARNOULT, M. D. The quantitative study of shape and pattern perception. Psychological Bulletin, 1956, 53, 45 2-457.

BERLYNE, D. E. The influence of complexity and novelty in visual figures on orienting responses. Journal of Experimental Psychology 1958, 55, 289-296.

BURROS, R. H. The application of the method of paired comparisons to the study of reaction potential. Psychological Review, 1951, 58, 60-66.

HULL, C. L., FELSINGER, J. M., GLADSTONE, A. I., \& YAMAGUCHI, H. G. A proposed quantification of habit strength Psychological Review, 1947, 54, 237-254.

KIMMEL, H. D., BOICE, C., \& LECKART, B. T. Paired comparison scaling of reaction potential in two different experiments on habituation. Behavioral Research Methods \& Instrumentation, in press.

LECKART, B. T. Task specific decrements in the duration of attention. Psy chonomic Science, 1968, 9, 559-560.

MOSTELLER, F. Remarks on the method of paired comparisons: III. A test of significance for paired comparisons when equal standard deviations and equal correlations are assumed. Psychometrika, 1951, $16,207-218$.

SOKOLOV, Ye. N. Perception and the conditioned reflex. New York: The Macmillan Company (Pergamon Press), 1963. P. 11.

\section{NOTE}

1. Address: Department of Psychology, San Diego State College, San Diego, California 92115.

(Accepted for publication December 13, 1968.) 